\title{
A DESCRIPTION OF DRIFT CHAMBERS USED IN A FERMILAB EXPERIMENT
}

\author{
R. THUN, C. W. AKERlof, P. Alley, D. KOLTICK, R. L. LOVELESS ${ }^{\dagger}$, D. I. MEYER, M. ZUMBERGE \\ University of Michigan, Ann Arbor, Michigan 48104, U.S.A. \\ D. BINTINGER, R. A. LUNDY, D. D. YOVANOVITCH \\ Fermi National Accelerator Laboratory, Batavia, Illinois 60510, U.S.A.
}

W. R. DITZLER, D. A. FINLEY, F. J. LOEFFLER, E. I. SHIBATA and K. C. STANFIELD

Purdue University, West Lafayette, Indiana 47907, U.S.A.

Received 10 August 1976

We give a detailed description of the drift chamber system used in a charm search at Fermilab. All important aspects of design and performance are discussed.

\section{Introduction}

We describe here in detail the drift chamber system used in a charmed particle search $^{1}$ ). This search has been the first major experiment at Fermilab to rely entirely on drift chambers for particle tracking. Drift chambers were chosen over spark chambers and multiwire proportional chambers since they combine excellent resolution with high rate capability. Our experience also indicates that drift chambers can be highly reliable and convenient detectors.

We remark from the outset that our efforts in drift chamber development were guided entirely by experimental considerations. These included ease of construction and maintenance as well as performance. What follows is a description of the drift chambers and our experience with their construction, testing and use.

* Work supported by the U.S. Energy Research and Development Administration and the National Science Foundation.

$\dagger$ Present address: Fermi National Accelerator Laboratory, Batavia, Illinois, U.S.A.

\section{Drift chamber configuration}

The drift chambers described here have tracked particles in the two-arm magnetic spectrometer shown in fig. 1. The aim of the experiment has been to find charmed particles by observing a narrow $\pi \mathrm{K}$ signal in an effective mass spectrum. Initial measurements are described in ref. 1.

Thirty-two drift chambers comprising a total of 368 sense wires are distributed in boxes DC1-5 in the left and right arms of the spectrometer system. Table 1 summarizes the configuration of chambers and sense wires within these boxes. The wire orientation is denoted by $\mathrm{H}=$ horizontal, $\mathrm{V}=$ vertical, and $\mathrm{W}=14.04^{\circ}$ with respect to vertical. Particles are deflected by the magnets in the vertical plane and this deflection is measured by the $\mathrm{H}$-chambers. The Wchambers are used for making unambiguous matches of the horizontal and vertical projections of multiple tracks.

Three different cell sizes corresponding to sense wire spacings of $0.8^{\prime \prime}, 1.6^{\prime \prime}$ and $2.4^{\prime \prime}(2.03,3.05$ and $6.10 \mathrm{~cm})$

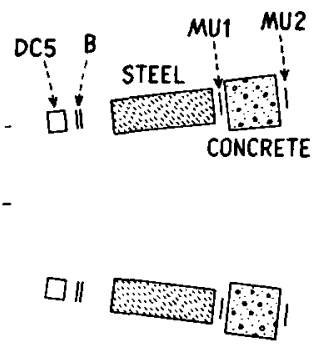

Fig. 1. View of the apparatus as seen from above. DC1-5 are the drift chamber boxes, C1-3 are Cherenkov counters and F, A, E, and $B$ are trigger counters. 
TABLE 1

Configuration of drift chambers.

\begin{tabular}{|c|c|c|c|c|c|}
\hline Module & Drift chamber & $\begin{array}{c}\text { Wire } \\
\text { orientation }\end{array}$ & $\begin{array}{l}\text { Number of } \\
\text { sense wires }\end{array}$ & $\begin{array}{l}\text { Sense wire } \\
\text { spacing (in.) }\end{array}$ & $\begin{array}{l}\text { Maximum drift } \\
\text { path }(\mathrm{mm})\end{array}$ \\
\hline \multirow[t]{4}{*}{$\mathrm{DCl}$} & 1 & $\mathrm{H}$ & 11 & 1.2 & 15.2 \\
\hline & 2 & $\mathrm{H}$ & 10 & 1.2 & 15.2 \\
\hline & 3 & $\mathrm{~V}$ & 8 & 0.8 & 10.2 \\
\hline & 4 & $\mathrm{~V}$ & 7 & 0.8 & 10.2 \\
\hline \multirow[t]{4}{*}{ DC2 } & 5 & $\mathrm{H}$ & 10 & 2.4 & 30.5 \\
\hline & 6 & $\mathrm{H}$ & 9 & 2.4 & 30.5 \\
\hline & 7 & V & 9 & 0.8 & 10.2 \\
\hline & 8 & $\mathrm{~V}$ & 10 & 0.8 & 10.2 \\
\hline \multirow[t]{2}{*}{ DC3 } & 9 & $w$ & 12 & 2.4 & 30.5 \\
\hline & 10 & W & 11 & 2.4 & 30.5 \\
\hline \multirow[t]{4}{*}{ DC4 } & 11 & $\mathrm{H}$ & 11 & 2.4 & 30.5 \\
\hline & 12 & $\mathrm{H}$ & 10 & 2.4 & 30.5 \\
\hline & 13 & $\mathrm{~V}$ & 10 & 0.8 & 10.2 \\
\hline & 14 & V & 11 & 0.8 & 10.2 \\
\hline \multirow[t]{2}{*}{ DC5 } & 15 & $\mathrm{H}$ & 23 & 2.4 & 30.5 \\
\hline & 16 & $\mathrm{H}$ & 22 & 2.4 & 30.5 \\
\hline
\end{tabular}

are employed to keep the number of wires per chamber reasonably constant. All chambers are arranged in pairs with one chamber shifted by half of a cell spacing with respect to the other. This pairing greatly simplifies the determination of the cell half through which the particle trajectory passed.

\section{Design and construction}

The chambers were designed according to one simple format independent of cell or chamber size. Every effort was made to simplify mass production as well as accessibility in case of repair. The basic chamber design is illustrated in fig. 2. Each drift chamber consists of a single frame of G-10 fiberglass. Holes are located at the four corners for mounting the frame on rods within the above mentioned boxes. Brass pins holding the sense wires and central field wires are seated in holes drilled into the sides of the frames. These holes are positioned accurately with respect to the four mounting holes and with respect to each other. The accuracy of locating the sense wires is at the 25-50 $\mu \mathrm{m}$ level. To achieve this accuracy, the hollow brass pins holding the central wires were capped with lead, the lead was milled to a smooth shape and a hole was then drilled into the lead with a diameter just slightly larger than the appropriate wire. (The sense wires are gold-plated tungsten with a diameter of
$25 \mu \mathrm{m}$ and the field wires consist of $127 \mu \mathrm{m}$ diameter hard copper.) With this arrangement it was easy to hand-thread the central wires into position and then solder them under tension to the outside ends of the pins. Moreover, since the pins holding these wires are held in place by set screws, defective wires were easily replaced.

The field-shaping wires on the outer surfaces of the drift chambers are soldered to circuit boards glued to the chamber frame. On one end a double-sided

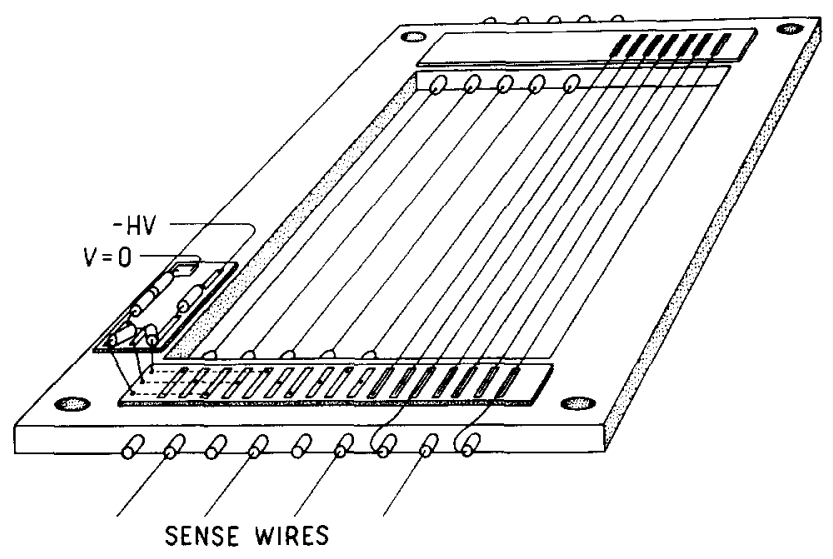

Fig. 2. View of a single drift chamber. For clarity field wires on the left and central wires on the right are omitted. 
circuit board with electrical feed-through connections is used to both hold the field wires and distribute the voltages generated by a resistive divider. The divider is located directly on the frame and consists of carefully matched resistors. The separation of field wires is $5.1 \mathrm{~mm}$ and the voltage gradient between adjacent wires is a constant $670 \mathrm{~V} / \mathrm{cm}$.

A cross-sectional view of a 2.4 " cell is shown in fig. 3 . The sense wire, at ground potential, is surrounded by a rectangular array of field wires. The voltage of the field wires decreases uniformly from $-1650 \mathrm{~V}$ near the sense wire to $-3700 \mathrm{~V}$ at the edge of the cell.

Sets of drift chambers are mounted inside aluminum boxes as shown in fig. 4. These boxes have openings, covered with Aclar windows, to keep free the active

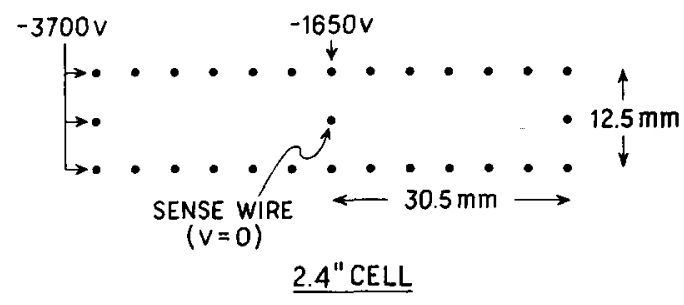

Fig. 3. Cross section of a single drift chamber cell and approximate operational voltages.

\section{DRIFT CHAMBER MODULE}

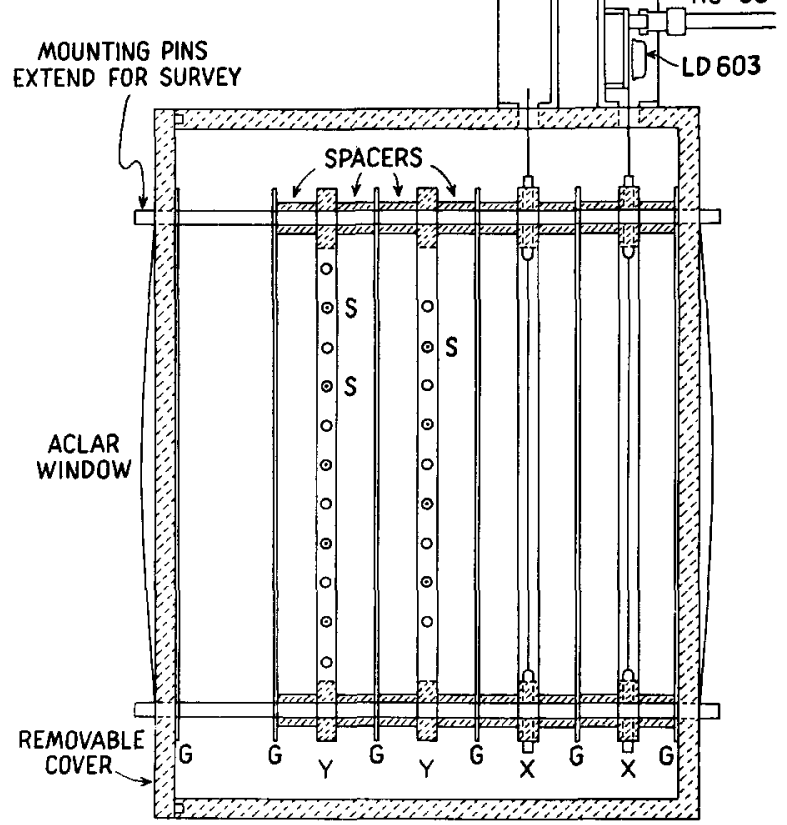

Fig. 4. Arrangement of drift chambers inside a box. $S$ are sense wires, $\mathrm{G}$ are ground planes, and $\mathrm{Y}$ and $\mathrm{X}$ are drift chambers for measuring vertical and horizontal coordinates, respectively. area of the chambers. The boxes perform several important functions: they provide a rigid, surveyable mounting for several drift chambers; they contain the drift chamber gas; and they shield the sense wires electrically from external noise sources. Aluminum foils at ground potential separate the drift chambers and cover the aluminum box windows. The foils act as clearing electrodes for the region between chambers and prevent ionization electrons from drifting into the cells from external regions. This is a critical point for a high-rate detector. The foils also complete the electrical noise shielding mentioned above. The sense wires are fed through the box to amplifier circuits enclosed in a covered aluminum U-channel. Shielded coaxial cables carry the output signals directly to the time-digitizing units.

To keep the chambers from binding on the mounting rods, the holes on the chambers have a bigger diameter than the rods. The difference is filled by an extension of the spacers separating the chambers and ground foils. Assembly or disassembly of the four chambers in a typical box has taken about fifteen minutes in practice. Changing a sense wire and associated pins has taken about five minutes.

\section{Choice of drift chamber gas}

After much deliberation we chose a mixture of $10 \%$ carbon dioxide and $90 \%$ argon as our drift chamber gas. This gas has a number of advantages. It is free of hydrocarbons which might polymerize and deposit on the drift chamber wires. (Presently, the exposure per sense wire has been about $10^{11}$ particles without noticeable degradation of chamber performance.) A side benefit of rejecting hydrocarbon mixtures is the elimination of fire hazards which are present for gasses such as isobutane. The argon- $\mathrm{CO}_{2}$ mixture can be vented directly in the experimental area. This mixture is also commercially available in accurate proportions $( \pm 0.1 \%)$ in high-pressure bottles. We have found that one half to one standard-sized gas bottle is sufficient to purge the air from all ten chamber modules and that a total flow rate of about one bottle every ten days maintained gas stability. (A lower flow rate might have been possible but was not tried.) The total volume of the ten drift chamber boxes is approximately $1 \mathrm{~m}^{3}$.

The drift velocity in argon- $\mathrm{CO}_{2}$ does depend on the electric field more strongly than it does in some hydrocarbon mixtures. Since it is impossible to maintain a constant electric field near the sense wire, small non-linearities are introduced in the relationship between drift time and drift path length. This presents 
no intrinsic problem as long as this relationship is stable in time. We have achieved such stability by fixing all operating voltages and using accurately mixed argon- $-\mathrm{CO}_{2}$. Details of the calibration procedures and results are described below. Except for saving some offline computing time, we see no particular reason for demanding absolute linearity of the drift chamber spacetime calibration. Other considerations, such as those mentioned above, are also important when considering a choice of gas mixtures.

\section{Electronics}

The entire drift chamber effort from initial development to final installation had to be and, in fact, was completed during a period of six months. We therefore decided to rely on available electronic components and devices rather than build our own.

As indicated in fig. 4, signals from each sense wire are carried to a LeCroy LD603 hybrid circuit mounted on the chamber module. The LD603 chip amplifies signals and produces output pulses of fixed amplitude and duration. The inputs to the LD603 are terminated with $100 \Omega$ resistors and the threshold is set to $0.4 \mathrm{mV}$. The output signals of the LD603 chips are transmitted directly to LeCroy Model 2228 octal time-to-digital converters (TDC) via 300 ns long RG-58 coaxial cable. The TDCs are started by a trigger formed from fast scintillator coincidence signals and stopped by signals from the sense wires. Such a "common start" mode accounts for the necessity of delaying the sense wire signals with a long coaxial cable. Although somewhat inconvenient, the common start mode has the advantage of digitizing the time interval corresponding to the first signal from a wire rather than that of an after pulse. Such after pulses occur at the few percent level. Of course, the long delay cable degrades the LD603 output pulses. We were thus forced to add the two complementary LD603 outputs by means of a fast transformer so as to produce a signal well above threshold at the TDC input.

This manner of digitizing drift times has been completely satisfactory for us. Clearly, only one particle per cell per event can be timed with this system, but in our view it is better to reduce cell size rather than attach more than one time digitizing channel per wire. During initial testing approximately two to three per cent of the LD603 chips failed. However, after this initial "burn-in" period we enjoyed four months of data-taking with only one chip failure out of 368 channels. The LRS 2228 TDCs were somewhat less reliable with about one unit failure per week. Installation of spares was relatively simple and little time was lost. Some of the TDC problems may have been caused by insufficient airconditioning in the counting room.

Drift chamber high voltages are provided by standard Fermilab power supplies ${ }^{2}$ ). These supplies are packaged in dual units with each unit supplying up to $800 \mu \mathrm{A}$ at a regulated voltage. For protection against breakdown, the power supplies have internal trip circuits. Pairs of identical drift chambers are attached to each power supply unit. No problems have been encountered in the use of these power supplies.

\section{Drift chamber problems and solutions}

In testing the drift chambers one is interested in determining their efficiency, resolution, calibration and stability of operation. These aspects will be discussed in detail in the following two sections. Here we would like to describe three serious problems that were encountered and solved during the initial operation of the chambers.

The first and most trivial problem was caused by the use of soft plastic Tygon tubing in feeding the argon$\mathrm{CO}_{2}$ gas mixture to the drift chamber boxes. The result was an insidiously slow contamination of the gas by the tubing. The effect manifested itself by a very slow but steady decrease of the drift velocity. We replaced the Tygon with hard plastic tubing (Imperial Eastman Poly-Flo) and no further contamination was observed.

A second, unexpected problem was caused by the electrical coupling of all cells within a chamber and one unfortunate property of the LD603 amplifier chip. As indicated in fig. 2 all field wires within a chamber that have a common voltage are connected by a good conductor on the bottom face of the circuit board. All sense wires of a chamber have a weak capacitive coupling to these groups of field wires. As a result, whenever a normal negative signal is observed on one sense wire, prompt positive signals of the same shape are observed on all other sense wires. The amplitude of these positive pulses is about ten times smaller than that of the negative signal pulse. The LD603 can be triggered by pulses with negative slopes even at positive voltage levels. Hence, when the negative signal pulse is sufficiently large, the other sense wires will trigger their chips on the trailing edge of the positive induced pulses. This undesirable trait was reduced to the point of insignificance by adding $100 \mathrm{pF}$ capacitors between the groups of field wires close to the sense wires and ground potential. 
The third and most serious problem encountered during initial operation of the chambers was the generation of self-sustaining discharges after exposure to intense radiation. The source of this problem was found to be a thin layer of non-conducting material on some field-shaping wires. Positive ions collect on these layers without being immediately neutralized. Strong, local electric fields are thereby generated which pull individual electrons out of the field wires. Some of these penetrate the insulating layer and drift toward the sense wire and produce an avalanche which supplies more positive ions to the defective field wire. The process is thus self-sustaining. An effective but depressingly laborious solution to the problem was found by cleaning all field wires with a non-metallic abrasive material (Scotch-Brite).

We are not completely certain of the origin of the non-conducting material on the field wires. During construction these wires did come into contact with human hands and material from the skin was probably not adequately removed from the wires by our cleaning methods. We consider this the most likely source of our problem. However, on the basis of our experience, we are not fully convinced that this was the only source.

\section{Calibration procedures and results}

We have calibrated the drift chambers using three independent methods. Two of these are based directly on tracking data obtained with all chambers fixed in place. The third method makes use of small test chambers that are moved across a narrow beam. These methods are now described in greater detail.

The first procedure makes use of the fact that the drift-time distribution is equivalent to the drift speed vs time distribution whenever there is a uniform particle flux across the cell. In our experiment this condition is approximately satisfied. To find the drift path length $x(t)$ corresponding to some drift time $t$ one simply integrates the time distribution $N(t)$ :

$x(t)=x_{\max } \int_{0}^{t} N(t) \mathrm{d} t / \int_{0}^{t_{\max }} N(t) \mathrm{d} t$,

where $t_{\max }$ is given by particles passing through at the edge $\left(x=x_{\max }\right)$ of a cell. The quantity $x_{\max }$ is, of course, equal to one half the cell size. Since the calibration is performed at the nominal (high) beam intensity one has to take precautions that random hits do not distort the time distribution $N(t)$. The effect of randoms is eliminated by finding tracks and rejecting from $N(t)$ all hits which correspond to a random particle passing through the cell at some earlier time. One final overall scale adjustment is required to correct empirically for the slight non-uniformity of the particle flux through the cells and for possible small errors in the determination of $t_{\max }$. This correction is actually quite small $(\sim 3 \%)$ and does not invalidate the basic idea behind this calibration method. The calibrations
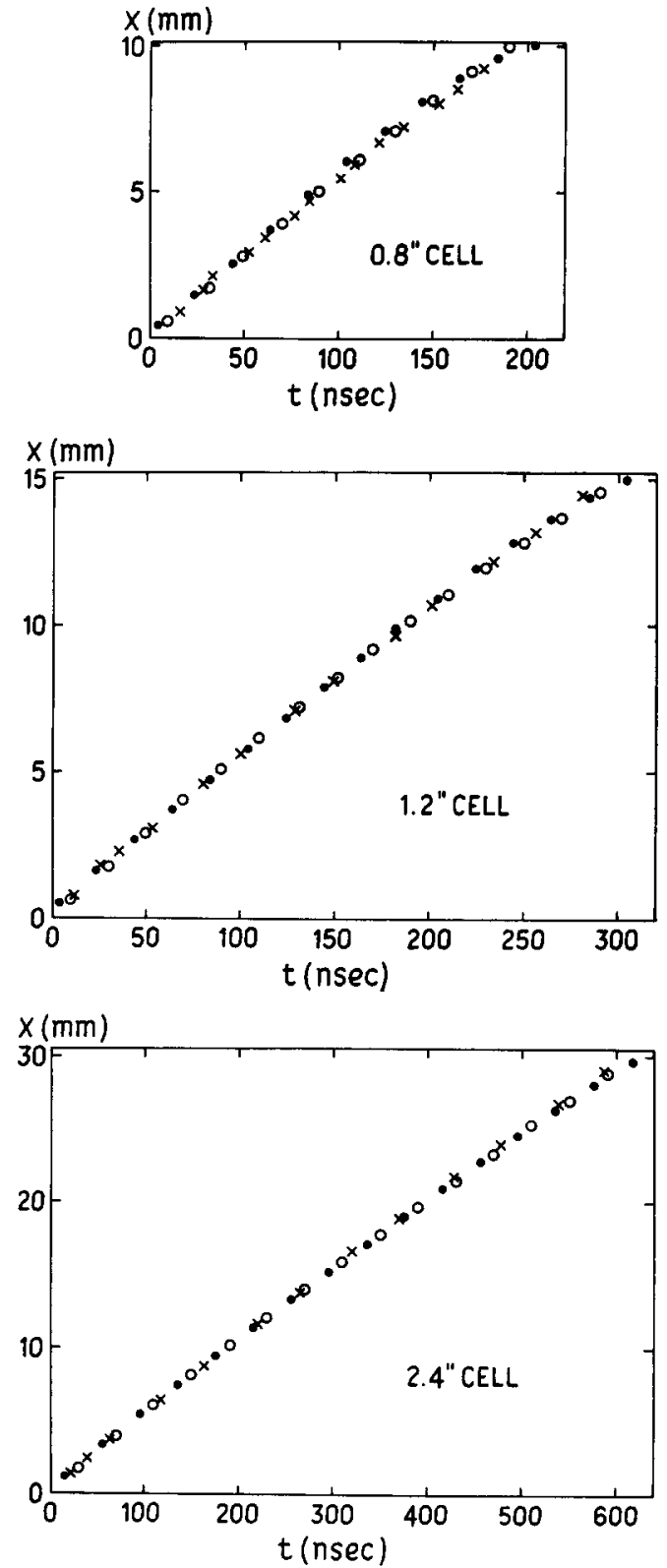

Fig. 5. Calibration curves for the three different cell sizes. Results from three different calibration procedures are given as follows: $O$ and empirical in-place calibrations described in text, $\times$ calibration with narrow beam and a movable test chamber. 
thus obtained are indicated by the open circles in fig. 5 .

The second calibration method is somewhat more general in that it does not assume any specific flux distribution across the cell. Like the first method, it also has the advantage of extracting the calibration directly from data runs with the chambers fixed in place. This method makes use of the fact that all chambers come in pairs shifted by half a cell width. For a particle that traverses such a pair of chambers with a reasonably small angle of incidence $\theta$ the following constraint applies:

$x\left(t_{1}\right)+x\left(t_{2}\right)=x_{\max }+d \tan \theta$,

where $x_{\max }$, as defined earlier, is equal to half the cell size and $d$ is the distance between the pair of chambers. A convenient way of finding the dependence of $x$ on $t$ is to parametrize $x$ as a polynomial in $t$, namely $x(t)=\sum_{n} C_{n} t^{n}$. The calibration constants $C_{n}$ are then found by fitting a large sample of events by the least-squares method to the above constraining equation. The angle of incidence $\theta$ is found for each event by tracking particles through the spectrometer. Results from this second calibration procedure are shown by the solid points in fig. 5 .

We have checked these convenient "in-place" calibrations by actually moving a set of test chambers with the three cell sizes across a narrow beam and measuring the drift time directly as a function of displacement. Such a narrow beam was defined by the traversal of $400 \mathrm{GeV}$ protons through a $1 \mathrm{~mm}$ wide scintillation counter near the test chamber. The measured relationship of drift time and drift path length is indicated by the crosses in fig. 5 .

As can be seen all three calibration methods give consistent results. Moreover, we have found the calibrations to be sufficiently stable in time, that only one set of time-independent calibration constants has been necessary for the duration of the experiment. All drift cells of a given size share a single calibration which gives the length of the drift path as a third-order polynomial of the measured drift time.

\section{Performance: efficiency, resolution, reliability, results}

We now turn to the important subject of how well the chambers have actually performed in the experiment. We begin with a discussion of chamber efficiency. Fig. 6 shows the efficiency of a 2.4" cell as a function of the voltage between the sense wire and nearest field shaping wire. This plateau was obtained with an electron source. The arrow indicates the voltage setting chosen for the experiment. The efficiency when the particle flux is reasonably low is at the $99 \%$ level. Under actual experimental conditions the definition of efficiency becomes more complicated. The reason for this is the very high particle flux incident on the chambers, especially those before the analyzing magnets. There, the single rates per sense wire are as high as 500000 per second. At those rates the primary loss of trackable coordinates is due to random particles which cross a cell and arrive at the sense wire before the signal of interest. For example, in the $2.4^{\prime \prime}$ cells if a particle passes through with the maximum drift time of about $600 \mathrm{~ns}$, then a singles rate of $0.5 \mathrm{M} \mathrm{Hz}$ yields a $30 \%$ probability of losing the coordinate of this particle. This is clearly the worst case in the experiment since the particles of interest have an average drift time in the large cells of about $300 \mathrm{~ns}$, giving a $15 \%$ loss rate at the highest random rates. The $0.8^{\prime \prime}$ and $1.2^{\prime \prime}$ cells are more immune to randoms because of their smaller maximum drift time. The pairing of chambers, with one shifted by half a cell size with respect to the other, provides strong protection against losing tracks to randoms. Clearly, the probability of losing a coordinate is greatest when a large drift time is involved. However, if the drift time is large in one cell it will be small in the other cell of the pair and thus quite safe from random loss there. The question remains whether or not the high particle flux also affects the electrical efficiency of the chambers. This can be answered by observing the rate of coordinate loss when no randoms are involved. The result is that, at the highest rates, the efficiency drops to about the $97 \%$ level.

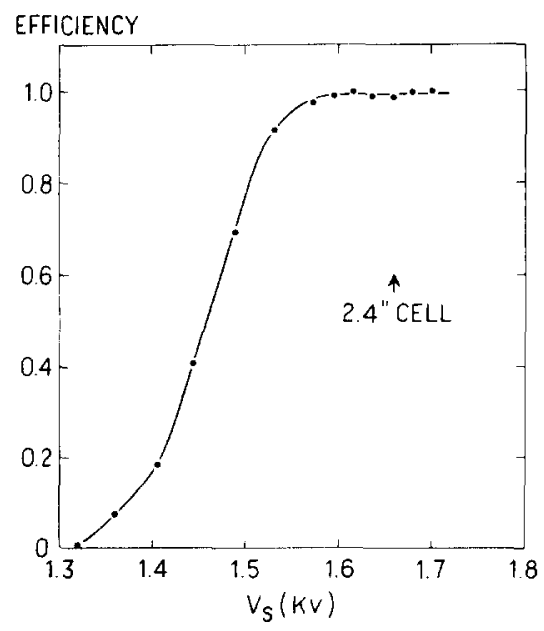

Fig. 6. Drift chamber efficiency as a function of $V_{\mathrm{s}}$, the voltage between sense wire and nearest field wire. The $0.8^{\prime \prime}$ and $1.2^{\prime \prime}$ cells exhibit similar plateaus. 
Pairs of adjacent chambers provide a direct measure of the intrinsic resolution of the coordinate measurement. Fig. 7 shows a distribution of the difference of the coordinate measured in one chamber and the coordinate measured in the other chamber extrapolated to the same position on the particle track. The intrinsic resolution is equal to the variance of this distribution divided by $\sqrt{ } 2$. The value obtained for the $2.4^{\prime \prime}$ cells, whose difference distribution is shown in fig. 7 , is $\pm 0.22 \mathrm{~mm}$. The other chambers yielded similar resolutions of around $\pm 0.25 \mathrm{~mm}$. The contribution of intrinsic chamber resolution to the spectrometer mass resolution is completely negligible compared to multiple scattering effects. We have therefore made no attempt to improve the resolution by calibrating drift chamber cells on an individual basis and have

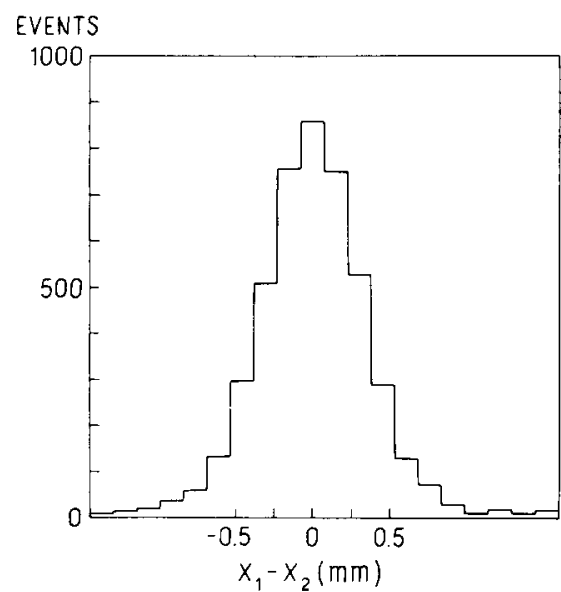

Fig. 7. Difference in coordinates measured by pairs of adjacent drift chambers. This curve yields an intrinsic resolution per chamber of $\pm 0.22 \mathrm{~mm}$.

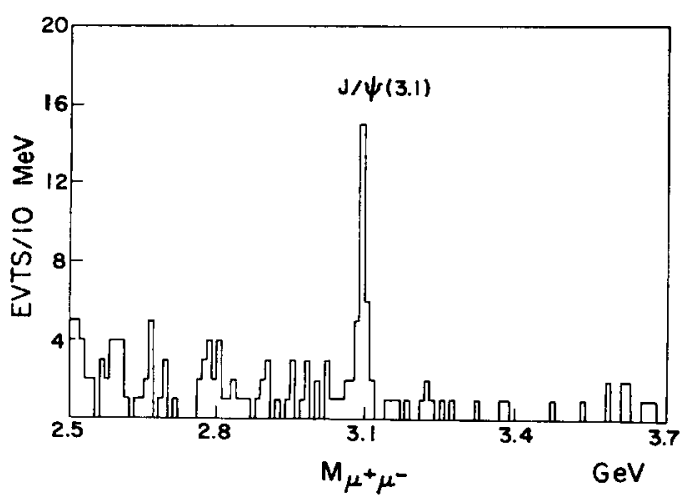

Fig. 8. $J / \psi(3.1) \rightarrow \mu^{+} \mu^{-}$signal observed with the drift-chamber spectrometer. continued to use one and only one set of calibration constants for all cells of given size.

We turn next to the question of reliability. So far, the chambers have experienced the equivalent of about three monhts of continuous experimental running in a very high flux environment. The exposure per sense wire has been about $10^{11}$ particles. During this time there have been no electrical or mechanical chamber failures: no broken sense or field wires, no resistor no electrical breakdown. Only one amplifier chip has failed and was quickly replaced. As mentioned earlier, the time-to-digital converters gave some problems but were basically quite reliable. In short, the chambers have been a pleasure to use and we hope that they will continue to perform as well in the future as they have in the past.

As an example of the results that can be obtained with a drift-chamber spectrometer, we show in fig. 9 the $\mu^{+} \mu^{-}$spectrum obtained during the initial period of our experiment. There is a clear narrow $J / \psi(3.1)$ signal evident in this spectrum. In fig. 9 we indicate the topology of one $J / \psi(3.1)$ event within the apparatus. Sense wires are shown as dots and those that gave

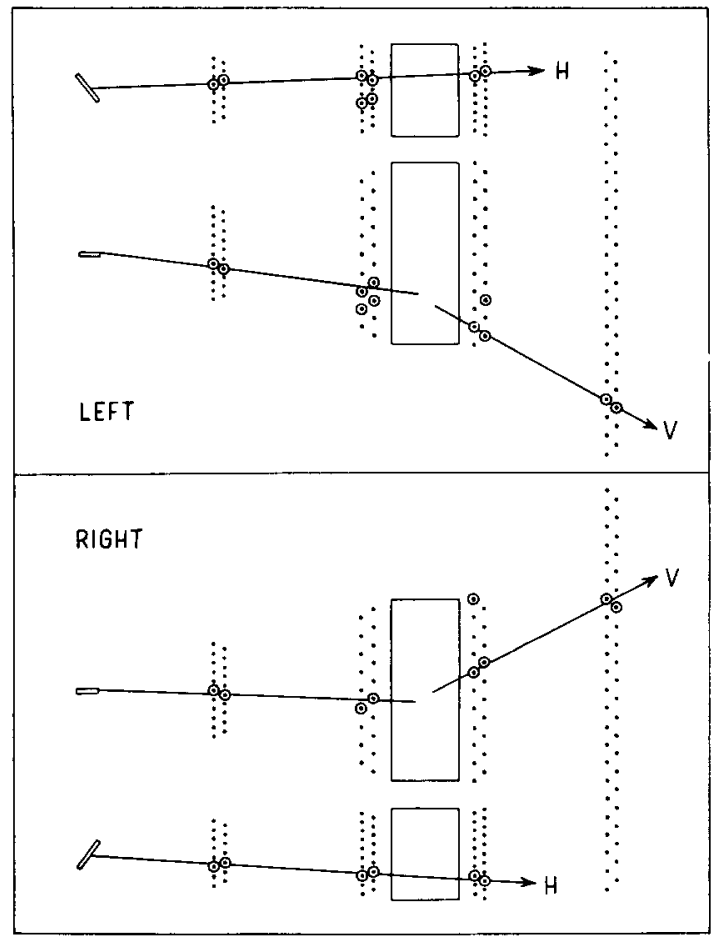

Fig. 9. $J / \psi(3.1) \rightarrow \mu^{+} \mu^{-}$event. The horizontal (H) and vertical (V) projections of the muon tracks in the left and right arm are indicated. Sense wires are shown as dots. Those with hits are circled. 
TABLE 2

Summary of drift chamber characteristics.

Number of drift chambers

Number of sense wires

Sense wire spacing

Sense wire

Field wires

Field wire spacing

Field wire voltage gradient

Half thickness of cell

Voltage between sense and nearest field wire

Gas

Drift velocity

Amplifier and pulse shaping

Time to digital converter

Average spatial resolution

Length of longest sense wire

Maximal normal singles rate per wire

Total cost per sense wire
32

368

$2.03,3.05$ and $6.10 \mathrm{~cm}$

$25 \mu \mathrm{m}$ diameter gold plated tungsten

$127 \mu \mathrm{m}$ diameter hard copper

$5.1 \mathrm{~mm}$

$670 \mathrm{~V} / \mathrm{cm}$

$6.3 \mathrm{~mm}$

$1650-1700 \mathrm{~V}$

$(10.0 \pm 0.1) \% \mathrm{CO}_{2},(90.0 \pm 0.1) \%$ argon

$5.0 \mathrm{~cm} / \mu \mathrm{s}$

LRS LD603 hybrid chip

LRS Model 2228 TDC

$\pm 0.25 \mathrm{~mm}$

$71 \mathrm{~cm}$

$0.5 \mathrm{MHz}$

Approximately 200 dollars signals are circled. The analyzing magnets are indicated by the rectangles between the central drift chamber units. The W-chambers are not shown in these vertical and horizontal projections.

\section{Summary}

We have summarized the most important aspects of the drift chamber system in table 2 .

We thank Dr. M. Atac for many interesting discussions during the development of our drift chambers. We are particularly grateful for the outstanding support of the Michigan and Purdue instrumentation and technical staff during the construction phase of the experiment. We also thank members of the Fermilab staff for their support of this effort.

\section{References}

1) D. Bintinger et al., A search for new particles in protonnucleus collisions at $400 \mathrm{GeV} / c$, Proc. 2nd Int. Conf. at Vanderbilt Univ. (1976).

2) T. Droege, Fermilab Technical Memo TM-527. 